\title{
A Function Oriented Corporate Network
}

\author{
P. S. - C. Wang and S. R. Kimbleton
}

ITT Programming, 1000 Oronoque Lane, Stratford, CT 06497

\begin{abstract}
The ITT/NET is an internal corporate network built to satisfy the distributed programming requirements of ITT technology and engineering communities. This paper describes the network: its goals, its architecture, its operational environment and prototype development activities.

The various issues involved in the construction of a Corporate Network based upon diverse, existing systems and communication methods are described in detail. The architectural and implementation decisions are discussed and justified. The programming process and the methodologies adopted are presented and some conclusions are drawn as to the practicality and usefulness of formal development methods in the context of this particular project.
\end{abstract}

\subsection{INTRODUCTION}

ITT is, perhaps, the preeminent example of a multi-product, multi-national corporation. Managing ITT requires planning, organization, communication and control. This requires effective communications within and between sites. ITT/NET is a corporate network designed to usefully interconnect the geographically distributed hardware, programming and human resources of major ITT North American and European units.

This report is a description of the ITT/NET. It concentrates on those aspects of the network as seen by the end user, that is, network services--their selection, their functions and functional relationships and their design and implementation. Service selection is summarized in Section 2, followed by discussions of network and protocol structures (Section 3) and the programming process: specification, design, implementation and quality assurance (Section 4).

\subsection{Distributed Programming Requirements and the ITT/NET}

It was early recognized that an application- and service-sharing network is needed to meet the computing requirements of major ITT technical activities. In response to this need, front end processors, leased lines and telecommunications packages were installed on several IBM mainframes

Permission to copy without fee all or part of this material is granted provided that the copies are not made or distributed for direct commercial advantage, the ACM copyright notice and the title of the publication and its date appear, and notice is given that copying is by permission of the Association for Computing Machinery. To copy otherwise, or to republish, requires a fee and/or specific permission. and a network started to evolve. However, with the growth of a major multi-national digital switch project (System 12, see [1] and [2]), problems concerning both the level of service and functional deficiencies of the network became critically important. ITT/NET is designed to solve these problems. It is a function oriented computer network designed primarily to interconnect geographically dispersed IBM mainframes, and secondarily, to connect IBM mainframes and other machines such as the DEC VAX and PDP 11 . It provides the functional building blocks underlying the distributed programming environments needed during the multi-location product life cycle.

\subsection{Network Services}

The service selection process was guided by analysis of the technical, managerial and system support activities for the afore-mentioned System 12 project, which resulted in the following communication requirements:

R1. Access to interactive applications on remote mainframes (a Terminal Access Protocol),

R2. Access to the batch processing facilities on remote mainframes (a Distributed Job Entry service),

R3. Transfer of data between locations (a File Transfer Protocol),

R4. Development, distribution and maintainence of person-to-person computer based information among sites (an Information Transfer Protocol),

R5. Shared access to a variety of programming management and support functions such as fault report and change control systems, and

R6. Support of network monitoring, operations, management and billing.

A further requirement is that the resources and the operations of the mainframe hosts should be controlled and managed by the individual hosts without being affected, in any way, by their participation in ITT/NET.

One well-known technical solution [3] to the above requirement is to offload communications support onto a separate computer (referred to as the Network Interface Machine, or NIM, hereafter), which deals with individual hosts on behalf of the ITT/NET user. This minimizes the network's impact on the existing mainframes and, at the same time, maximizes its ability to accomodate to constant changes in network configuration (e.g., the connection of a new host to the network), and func- 
tions (e.g., the addition of a new File Transfer feature). This is the approach we have chosen to adopt.

Interfacing to IBM systems is further complicated since any impact or perturbation to existing IBM program product or application environment must be avoided if at all possible. Of the several possible approaches, Remote Job Entry (e.g., HASP [4]) emulation is probably the most popular. In this design, the network interfaces perfectly with the mainframe IBM program products and requires no change whatsoever in the mainframe's mode of operation. However, it does impose a transaction style of transport between the network (i.e., NIM) and the mainframes. This point is further discussed in Section 3 below.

\subsection{Network Configuration}

Each mainframe is connected to ITT/NET through the NIM, a local IBM 4300. The complete network is formed by interconnecting these NiMs through leased communication lines. supplied by the various telephone authorities. The physical configuration of ITT/NET is given in Figure 1. Characteristic programming configurations for the host and the NIM are shown in Figure 2.

\subsection{FUNCTIONAL ARCHITECTURE}

Experiences derived from existing networks indicate clearly that the provision of user-oriented protocols such as those given above require a new protocol level to be developed. It is no longer practical to view the communication facility as a simple data pipe which can be tapped at the two ends and used for a specific application. Rather, a standard programming facility needs to be established to handle host-to-host communications. That is, in addition to the protocols listed in Section 2.1, we need a data transport mechanism, which, for ITT/NET, is called the Universal Transport Protocol (UTP), providing

R7. A program-invocable transport or end-to-end service allowing transparent data transfer among NIM applications and between NIMs and mainframes.

This collection of functions, taken together, covers the uppermost three layers (4-7) of the ISO Model for Open System Interconnection [12], and corresponds to what is commonly referred to as High Level Protocols (HLP).

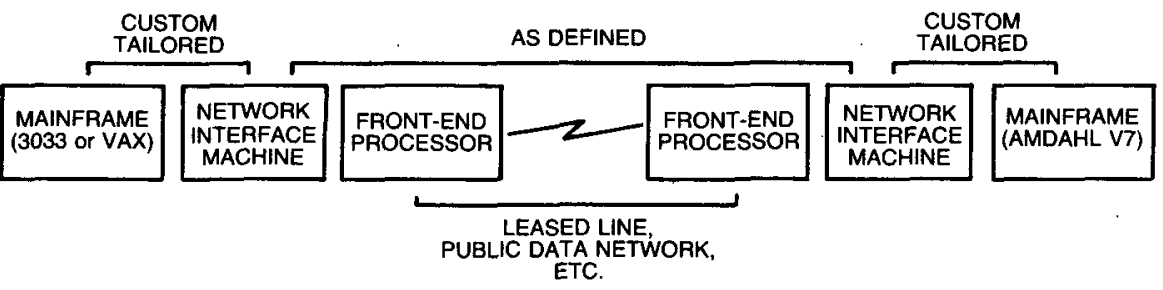

FIGURE 1. ITT/NET Network Configuration

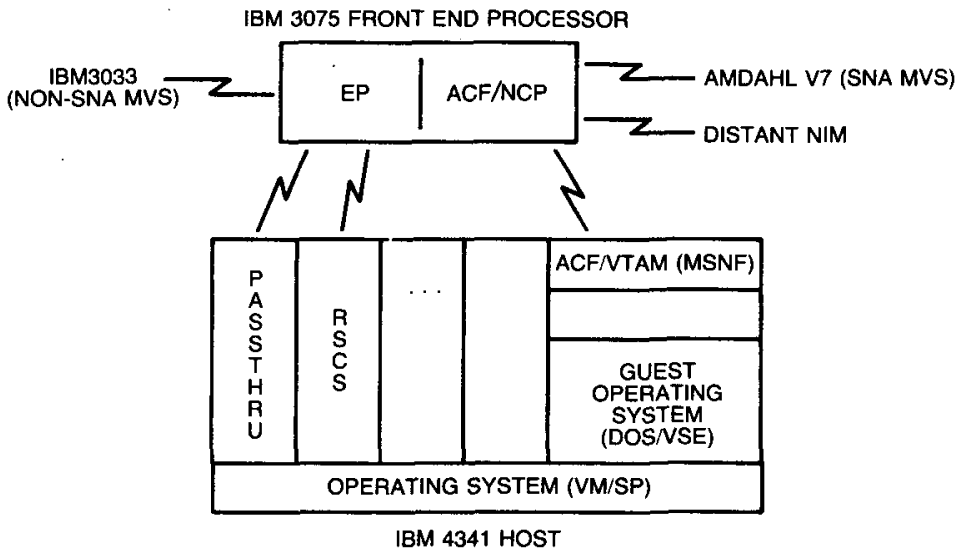

FIGURE 2. NIM PROGRAMMING CONFIGURATION

The mainframes ("hosts") are connected to NIMs, which are in turn interconnected to form a multi-domain, SNA network [5]. The NIMs handle both IBM SNA and non-SNA based hosts, e.g., IBM 370 s under the MVS operating system with Virtual Telecommunications Access Method (VTAM) [6] and DEC VAX under VMS [7] with HASP emulation. This is achieved by running both SNA and non-SNA communication packages on NIM: VTAM in a guest operating system and Remote Spooling Communications Subsystem (RSCS) [8] as shown in Figure 2 below. (See [9], [10] for further details of the NIM hardware and programming configuration.)

\subsection{ITT/NET Hierarchy}

As the above discussion indicates, the ITT/NET protocols are organized into a hierarchy of many levels ${ }^{1}$ : each level uses the functions of the lower level, and adds some function of its own for possible use by the next higher level.

There are four levels of interest in the ITT/NET (see Figure 3), but there may be more than one protocol within a level, representing different uses of the lower levels and different services to the 
upper levels. The lowest level of the hierarchy consists of vendor communication packages, which provide the basic data transmission facilities (the communications subnetwork). The next level specifies methods for the origin NIM application to transfer (logical) messages to a destination NIM or mainframe application. This protocol, the ITT/NET Universal Transport Protocol, is used by all higher level services (see Section 2.1) as their common host-to-host communication vehicle.

At the next higher level are the building block protocols which provide general support services (file access, remote job entry, terminal access and message transfer) for the networked environment. These are also the foundation on which the next and highest level, multi-location development protocols are built to serve application specific aspects of communications between remote program development support applications.

A diagrammatic illustration of this protocol hierarchy is given in Figure 3 below, together with the corresponding ISO numbers for these levels. tional partitioning among protocols but also forms the framework for design modularization (see Section 3.2 for further discussions).

\subsection{Realizing the ITT/NET Hierarchy: Structural Layering}

The ITT/NET protocols are implemented on an IBM machine running the VM/SP system. This operating system manages the hardware machine in such a manner that many virtual machines appear to exist.

Each user, when s/he logs onto the system, has at his/her disposal a "virtual machine" running the (single-user) conversational operating system CMS. In addition to CMS commands and utilities, users are provided the set of ITT/NET specific commands or protocols described above. These user-level (building block) protocols perform the data processing and communications functions to support the networking needs of the users as well as to provide for the creation of more sophisticated, application specific communication services (a Multi-location Development Environment)

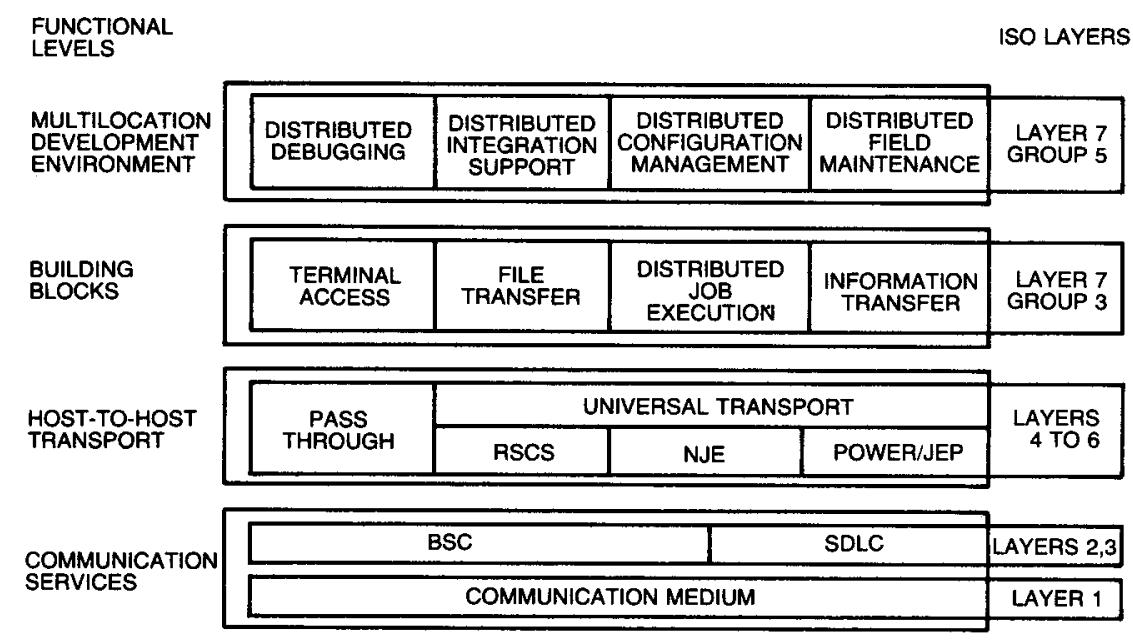

FIGURE 3. THE ITT/NET FUNTIONAL HIERARCHY

The advantages of architectural layering are well known: it promotes manageability and layer independence through functional grouping and localization. This is particularly important for the ITT/NET for the following reasons. First, it gives us the flexibility to replace level functions with equivalent ones without affecting other levels in the hierarchy. Secondly, it provides a framework upon which new protocols can be developed. Thus the two critical growth areas for ITT/NET--improvements to current protocols and construction of distributed applications--can be planned and implemented in an orderly fashion. Thirdly and most importantly, given the NIM programming base, layering not only provides func-

2 We purposefully use the term "level" instead of the ISO "layer", to emphasize the difference between our scheme and that of the Open System Architecture. In particular the numbering of our levels is entirely arbitrary although the choice of points at which to divide trie levels is guided--at least in principle--by the CSI model.
In each user-level protocol there are three logical components (see Figure 4), each distinguished by the kinds of operations it performs:

User Support Component. USC. A component that interprets user commands, accesses user's files, and in general performs operations in direct support of the user.

Protocol Support Component. PSC. A component that performs operations needed to support the functioning of the protocol, but not directly visible to the user: buffering and file attribute--record format, record length, etc.--handling are examples of the PSC functions for file transfer protocols.

Transport Support Component. TSC. A component that transfers information from one place in the network to another by invoking and interfacing with the host access method wusuch as VTAM or RSCS.

The functional layering given in Section 3.1 interact strongly with the above structural decompos- 
ition. As shown in Figure 4 the lowest level (level 1) of the ITT/NET hierarchy is composed of vendor packages such as VTAM, providing basic communication facilities. Thus this level covers the TSC-to-TSC portion of the HLP top-level structure ${ }^{2}$.

Level 2 (UTP) erases network (SNA vs. non-SNA) boundaries to provide a "universal" transport service. The UTP packages the data from these buffers and uses Level 1 to transmit the data to the destination machine. UTP uses the SNA communication products in the NIM, in tandem with the non-SNA facilities such as RSCS networking [9]) to accomplish this. Structurally UTP is the TSC-to-PSC interface (as well as the pre- and post-processings associated with this interface, e.g., packaging of data into a form accepted by the TSC).
Top level design and test plan are formulated, and through step-wise refinement, the design is decomposed into modules and the logic and data flows that implement these modules. Also, detailed test plans are defined to validate the functional, performance and interface behaviors of the modules. The actual coding and testing (implementation phase) of these modules then follow, and the test results analyzed and documented.

The next phases (limited availability, general availability and termination) deals with the installation, support and withdrawal of the programs. Since our experience so far has been limited to the first four phases, we shall, in the following, confine our description of ITP programming process to this portion of the life cycle.

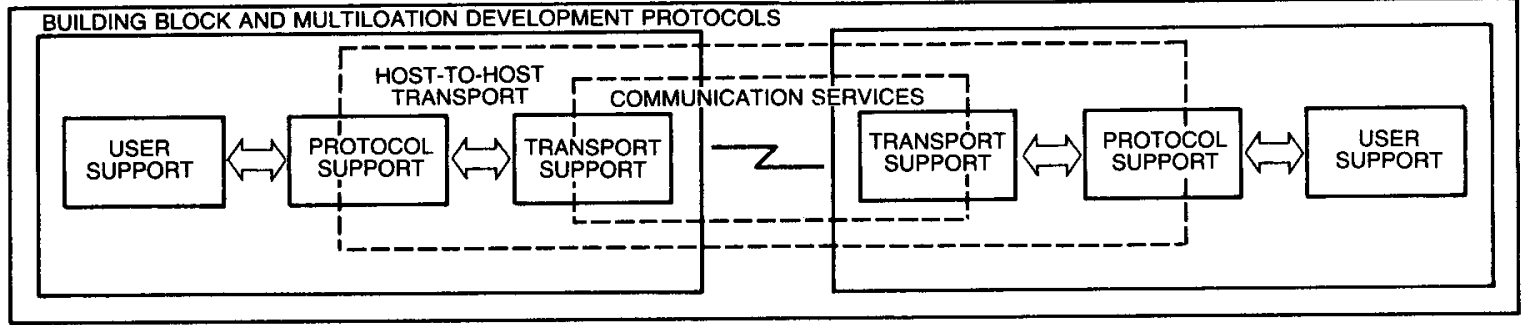

FIGURE 4. THE ITT/NET STRUCTURAL HIERARCHY

Thus, the hierarchical functional dependencies among HLPs map directly into the levels of "design layering", which, in turn, forms the basis for the top-level designs of all ITT/NET end-user protocols as will be discussed further in Section 4.

\subsection{Programming Process}

So far we have concentrated on our technical approach. Let us now turn to the ITT/NET programming process and describe the actual implementation of this approach. In the interest of concreteness, instead of dealing with the issue in general, we shall concentrate on one specific application, the VM/CMS implementation of the Information Transfer Protocol (ITP).

\section{1 Formalized Methodology}

As mentioned in Section 2, the first step we took is a System 12 communications requirements analysis. This, together with the objectives of early availability, extensibility and reliability provides the input from which the basic architectural framework (Section 3.2) and the functional specifications and the top level designs of individual protocols are derived. These activities constitute the first two phases of the programming effort - conceptual planning and definition.

The output of this phase, the functional specification, form the input to the next or, design phase.

2 However, this coverage is in parts rather than as a whole: the diversity of these packages and their lack of interworkability makes it impossible to treat the ITT/NET as one single, integrated network at this level.

\subsection{An Example - the Information Transfer Protocol}

As described in Sections 1 and 2, analyses of System 12 communication requirements discised the need of an information transfer service. Tnat is, a function that allows a VM/CMS user to create, edit, file and distribute memos, transparently and uniformly, to other CMS and VAX/VMS users. (An MVS interface is being implemented but will not be covered here since it remains to be completed.)

The ITP definition phase starts with a feature-by-feature comparison of existing information transfer facilities and user needs, in terms of command primitives, command parameters and their execution effects. This results in the extended list of message system primitives given in Figure 5 . The criteria of level of demand, implementation ease and sufficiency are then used to partition this list into three sets as shown in the Figure: a foundation or minimal set of basic services, a kernel set of essential features needed to support most near-term applications and a value-added set of desirable future enhancements.

Based upon this analysis and the goal of near-term usability, we selected the kerns: set for the initial implementation and proceeded towards its top-level design.

\subsubsection{The ITP System}

As user interface, we selected the the IBM System Productivity Facility for VM [13 $]^{3}$. This environment, and the design layering of Section 3.2, dictates that ITP operate as a reentrant SPF

3 . This is the environment used for System 12 development. 


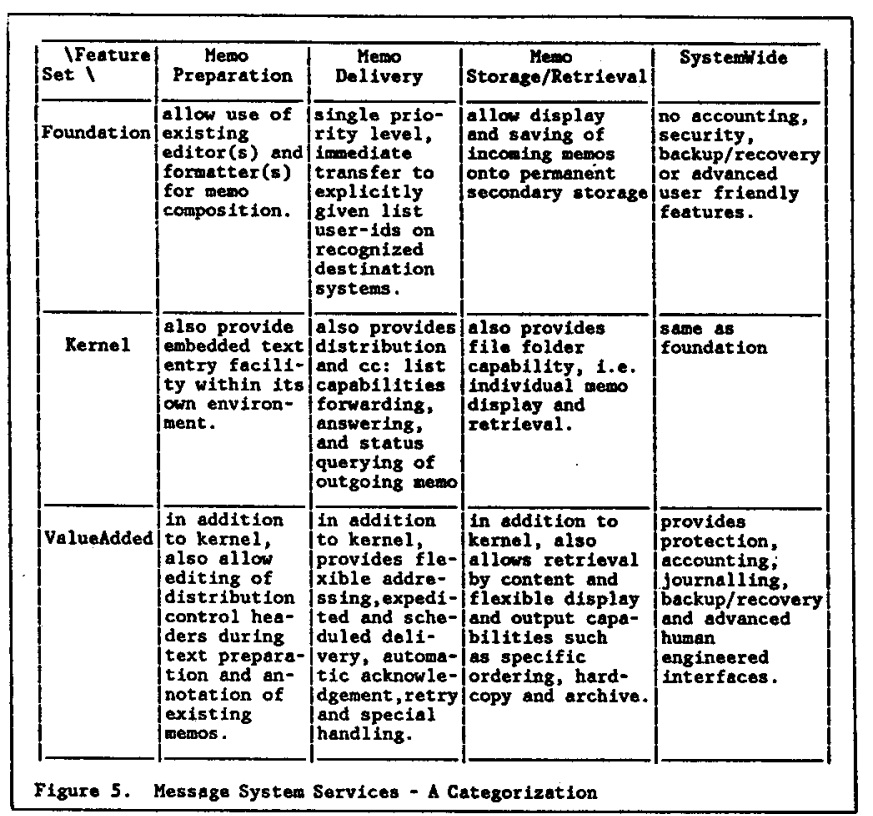

procedure, in the user area of the user's CMS machine and interfaces with UTP to effect communication with distant NIMs. The programs, files and modules constituting this top level ITP are given below (in PDL notation) [14]:

$\bmod$ ITP

programs ITPPRI

datasets INMEM, DRAFT, INBOX dom

modules ITPGTM, ITPSND, ITPREA, ITPINP

where INMEM is the CMS file containing the memo to be sent or read, DRAFT and INBOX are the default "mailbox" file for storing the (newly created) outgoing and incoming memos, respectively. The program ITPPRI is the the primary processor which checks pending incoming memos, retrieves and stores them in INBOX, and decodes user commands and calls the appropriate send and read modules for processing the selected commands.

The modules ITPGTM and ITPINP are common processing routines-routines invoked by other pro- grams rather than directly by users (i.e., from the command level).

Figure 6 is a (partial) illustration of these components and their functional relationship to SPF, UTP and the CMS file system. (The top-level design for the SEND module is given in Fig. for illustrative purposes. A complete description is available from [11].)

It is worthwhile to point out that at this top design level, we are primarily concerned with the functions and the functional relationships of the major ITP components (ITPPRI, ITPGTM, ITPSND and ITPREA) with each other and with the environment. Thus only the interfaces are specified fully (e.g., all possible user inputs are accounted for), whereas most of the processing details (e.g., how the memos are made up and how they are transferred to the recipients) are left unspecified.

The task of verifying the logical correctness of the design and that it satisfies the ITP service specification is approached from a formal ("verification") as well as an empirical ("testing") direction.

Formal techniques for protocol verification are still in its early development stage--so far they have only been used to verify a very limited set of "general" (i.e., applicable to all) protocol properties, and this is what we confined ourselves to ${ }^{4}$. In specific, we verified that ITP satisfies the following

Invariant Assertion [14], [15]:

All valid messages sent by the user (i.e., SEND requests addressed to recipients with valid USERID and NODEIDs) are delivered to the recipient.

Details of the proof are included in the appdendix for readers' reference. The main task--the demonstration that our design meets its specification is dealt with below, using the less rigorous but far more practical method of testing.

4 However, we did find that this exercise in mathematics invaluable in providing precision and rigor to the final design.

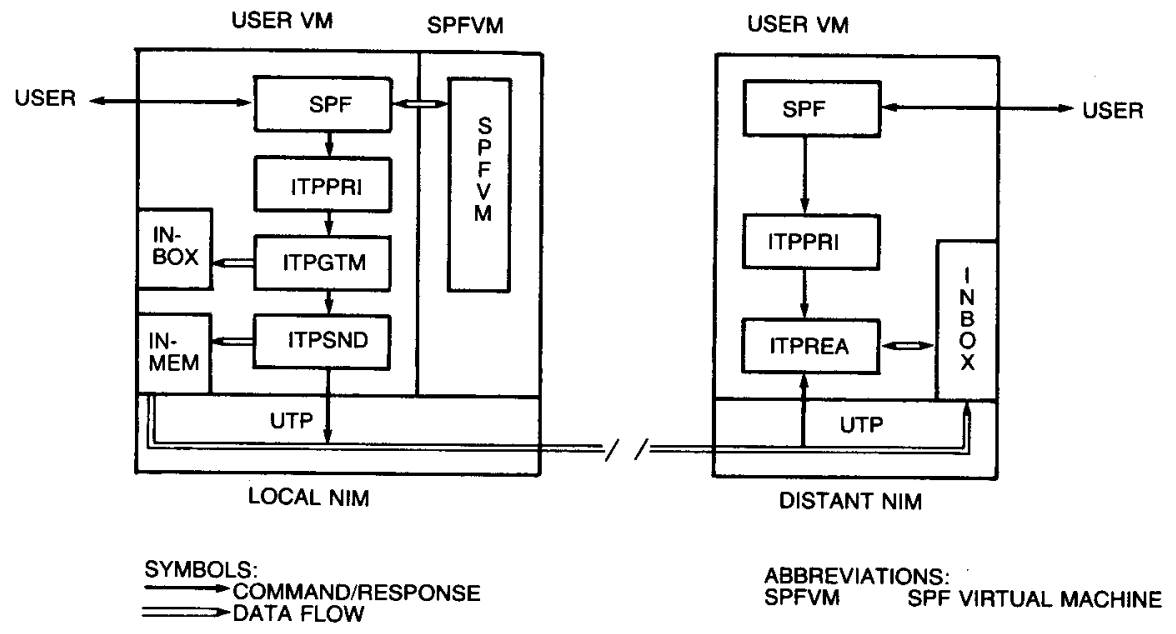

FIGURE 6. ITT COMPONENTS AND RELATIONSHIPS WITH SPF AND UTP 


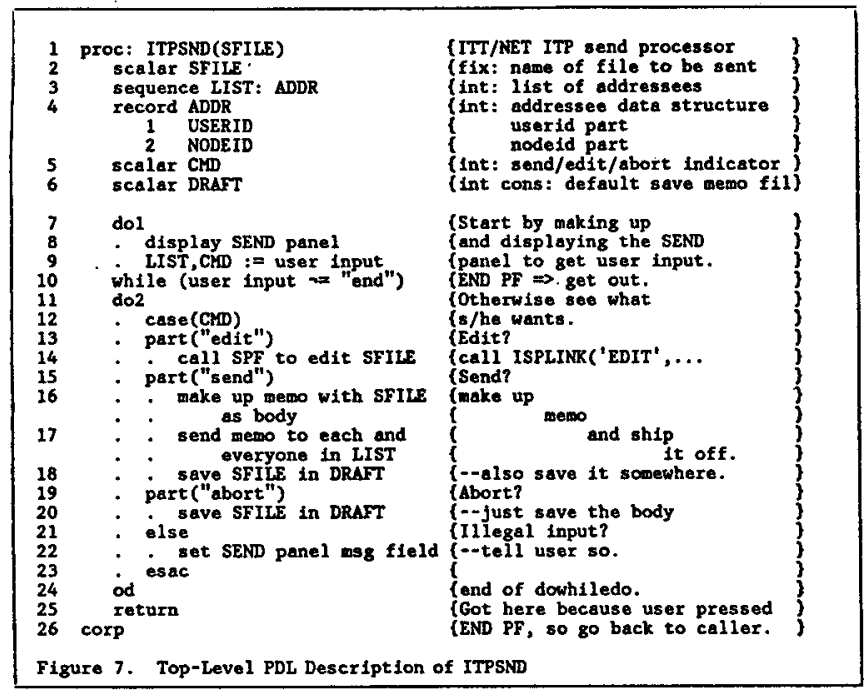

\subsubsection{Function Testing}

Top-level test cases are devised to detect deviations of protocol behavior from that prescribed by their specification. The basic strategy is exhaustive functional testing by

1. examining the service specification,

2. finding out the logical relationship between the inputs and outputs (inputs and outputs are defined as anything that flows between ITP and its environment)

3. finding all (environment or pragmatic) constraints that these input conditions and output consequences must satisfy and from this

4. deriving all possible input/output combinations and use them as test cases, and finally

5. analyzing these cases so all input and output boundaries are covered at least once.

\subsubsection{Module Organization and Module Testing}

The above design (Section 4.2.1) is further expanded and reorganized until a level of detail is reached that is appropriate for module specifications. The resulting detailed design is illustrated by the following module dependency chart and the PDL expansion of Figure 8 and Figure 9 below.

Test design, at this phase, is based upon logic coverage. That is, a set of test inputs are selected and the decision table representation of the modules are formulated to check that sufficient cases are covered so that all branches and statements are covered at least once.

This is followed by the actual implementation, that is, converting the detailed design into code and the carrying-out of the tests. In the case of ITP, PL/I was used for most of the coding but since a great deal of essential VM system services (e.g., file access) are not available directly to $\mathrm{PL} / \mathrm{I}$, assembler routines were also written for the interface functions.

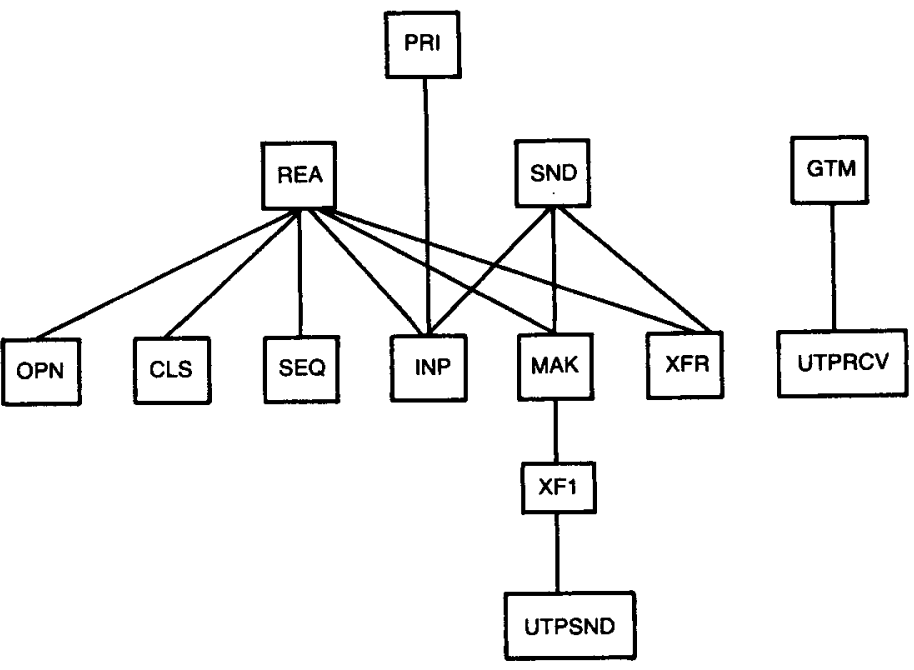

FIGURE 8. ITP MODULES AND MODULE DEPENDENCIES 
This completes our description of ITP services. But before turning to the next topic, a summary of current ITP status might be of interest:

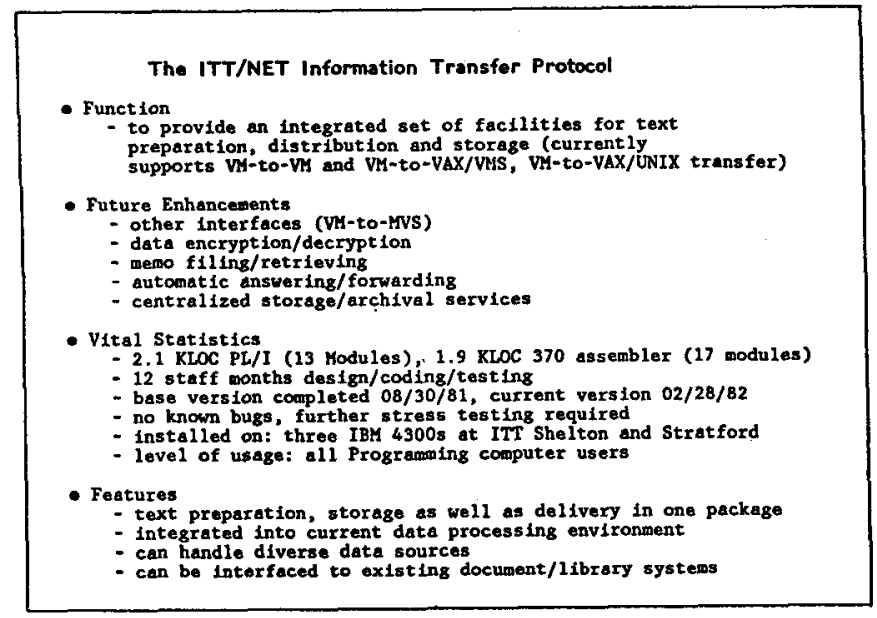

\subsection{Concluding Remarks}

The ITT/NET is a high-level, application-independent framework for the construction of distributed systems and programming development tools. It eliminates the need for application-specific communication protocols and support programming. The framework consists of a network-wide communications access method (the Universal Transport Protocol), and a set of utilities for file and job transfering (the File Transfer Protocol, the Information Transfer Protocol and the Distributed Job Execution function).

Since ITT/NET is built on top of existing operating systems and communications methods, considerable care was given to the structuring of protocols to achieve this "vendor-product" independency. This paper presents the crucial decisions in the structuring of the various protocols. We hope that the architecture were formulated with caution so the decisions we made will prove to be sound architectural choices--but only time will tell.

A second focus of this paper is on the programming disciplines and practices used to realize this architecture. Emphasis was placed upon methods based upon theoretical foundations such as formal proofs and testing techniques. Our experience so far is that formal methods are indeed practical and that they contribute to improved quality in significant ways: they bring in precision and order to the programming process, and provide a standard language during each development phase for communication among users, managers, designers and implementers.

\subsection{References}

[1] Cox, J.E., Lawson, D.A., Denenberg, J. and $\checkmark a n$ den Brande, F. A Digital Switch for Wide Range of Application. ICC'80 Conf. Rec. $(1980) ; 46.1 .1-46.1 .7$.

[2] Lawson, D.A. A New Software Architecture for Switching Systems, IEEE Trans. Comm., vol. COM-30 (1982), 1281 - 1289.

[3] Roberts, L.G. and Wessler, B.D. Computer Network Development to Achieve Resource
Sharing, AFIPS SJCC, vol.36 (1970), 543 549 .

[4] HASP Networking PRPQ: System Programmer's Guide. Order No. SH20-1981, IBM Corporation, Data Processing Division, White Plains, NY.

[5] Systems Network Architecture General Information. (1st Ed.) Order No. GA27-3102-0, IBM Systems Development Division, Research Triangle Park, NC (1975).

[6] Advanced Communications Function for VTAM General Information: Concepts.(2nd Ed.) Order No. GC27-0463-1, IBM Corporation, Data Processing Division, White Plains, NY (1980).

[7] VAX/VMS Primer. Order No. AA-D030A-TE, Digital Equipment Corp., Software Distribution Center, Maynard, MA (1978).

[8] IBM Virtural Machine/370: Remote Spooling Communications Subsystem Networking General Information. (2nd Ed.) Order No. GH24-5004-1, IBM Corporation, Data Processing Division, White Plains, NY (1980).

[9] IBM Virtual Machine/System Product: Introduction, Order No.SC19-6200. IBM Corporation, Data Processing Division, White Plains, NY.

[10] Kimbleton, S. R. and Wang, P. S.-C., ITT/NET Communication Support for Program Development. Electrical Communication, vol.57, no. 4 (1983), 320 - 325 .

[11] The ITT/NET Information Transfer Protocol. Internal Documentation, ITT Programming Technology and Development, 1000 Oronoque Lane, Stratford, CT 06497 (Aug. 1981).

[12] Data Processing - Open Systems Interconnection - Basic Reference Model. International Standards Organization, Draft Proposal iSO/DP 7498 (1980)

[13] Structured Productivity Facility for VM/SP Program Reference. (1st Ed.) Order No. SC34-2047-0. IBM Corporation, Data Processing Division, White Plains, NY (1981).

[14] Linger, R.C., Mills, H.D. and Witt, B.I. Structured Programming. Addison-Wesley Reading, MA (1979).

[15] Owicki, S.S. Specification and Verification of a Network Mail System. in "Program Construction", Lecture Notes in Computer Science, Springer-Verlag, New York, NY, vol.69 (1979), $198-234$ 
Appendix. Formal Correctness Proof for the ITT/NET ITP

In order to carry out the proof, auxiliary variables are needed to record the history of ITP memo processing: we shall use $H(M 1, M 2, r)$ to denote the sequence of messages passed from module MT to module M2 that has " $r$ " as its recipient". This enables us to restate the correctness assertion (Section 4.2.1) in terms of the history of messages passed between the user and ITP:

For every request and every valid user $u$ and recipient $r$, the assertion

$f=(H(u, I T P, r)=H(I T P, r, r))$ holds true.

We shall proceed by first analyzing the executior sequences of ITP programs using the E-chart and trace table techniques of [14]. From the results of this analysis and the

UTP Invariant Assumption: $\mathbf{g}=(H($ ITP , UTPSND, $r)=H($ UTPRCV $, r, r))$

we then deduce, using ordinary logic, that the above assertion in fact holds.

Proof:

From the PDL descriptions of the various ITP modules (see -- Figure 7 -- and [11] we identified four separate ITP paths (labelled path $1-4)$ in the (loop-free version of the) E-chart as shown in Figure 10 We now proceed to look at its behavior along each path (i.e., the trace table for that path) and show that the invariant assertion holds for each case.

For brevity, the letters $U$ and $R$ are used to designate the message histories $H(u, I T P, r)$ and $H(I T P, r, r)$, respectively. Each row of the trace table expresses the current value of the data items $U$ and $R$ (denoted as $U 1, R 1$ for the first row, U2, $\mathrm{R} 2$ for the second row, etc.) in terms of their previous values. The initial histories for messages passed from the user to ITP and from ITP to the recipient are represented by $H O(u, I T P, r)$ and HO(ITP, $r, r)$, respectively.

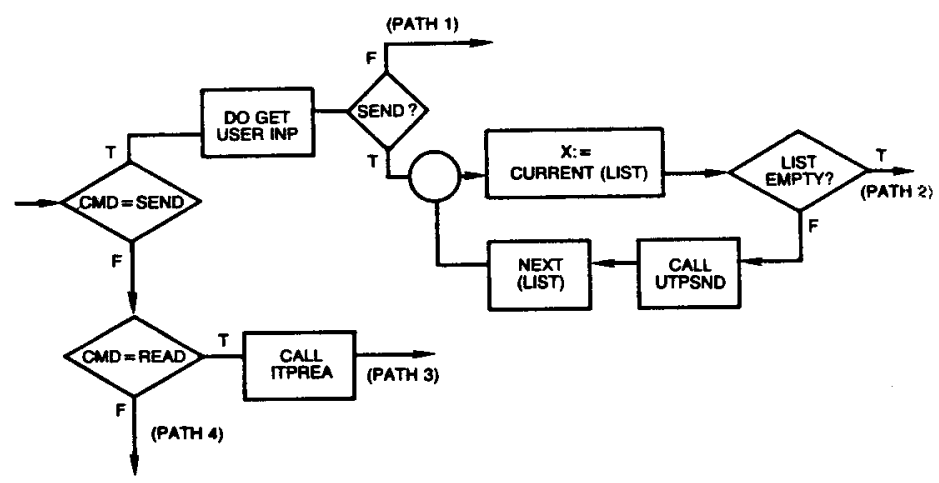

FIGURE 10. TP EXECUTION CHART

The notation as well as the discussion for our proof follows closely that of Linger (Ref. [14], Chap. 6], which should be consulted for details. function

$f=(H(u, I T P, r)=H(I T P, r, r))$

program (see Figure 10)

1 case (CMD)

2 part ("SEND")

3 . do get user input

4 . . if (user input $=$ "send")

5 . . then

6. . do1 $x:=$ current(LIST)

7. . while ( $x=$ empty)

8. . do2

9. $\cdot$. call UTPSND

$10 \cdot \cdot \cdot \cdot \operatorname{next}($ LIST)

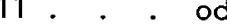

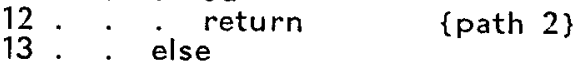

14 . od return \{path 1 \}

16 part ("READ")

17. call ITPREA

18 . return

19 else

20 . return

21 esac

\{path 4\}

proof

I. path 1 (SEND, abort)

\begin{tabular}{|c|c|c|c|}
\hline part & condition & $U(=H(U, \operatorname{ITP}, r))$ & $R(=H(I T P, U T P S N D, r)$ \\
\hline $\begin{array}{c}1 \\
3-7\end{array}$ & $\begin{array}{l}\text { CMD }=\text { SEND } \\
\text { abort }\end{array}$ & $\begin{array}{l}\mathrm{U1}=\mathrm{HO}(\mathrm{u}, \mathrm{ITP}, \mathrm{r}) \\
\mathrm{U} 2=\mathrm{LIST} \text { members } \\
\text { addressed to } \mathrm{r} \\
\mathrm{U}=\mathrm{U} 1\end{array}$ & $\begin{array}{l}\mathrm{R} 1=\mathrm{HO}(\mathrm{ITP}, \mathrm{UTPSND}, \mathrm{r}) \\
\mathrm{R} 2=\mathrm{R} 1 \\
\mathrm{R} 3=\mathrm{R} 2\end{array}$ \\
\hline
\end{tabular}

path function: $\quad H(u, I T P, r)=H(I T P, U T P S N D, r)$

This together with the UTP invariants (g) leads directly to the intended function $f$.

II. path 2 (SEND, -abort)

\begin{tabular}{|c|c|c|c|}
\hline part & condition & $\mathrm{U}(=\mathrm{H}(\mathrm{u}, \mathrm{ITP}, \mathrm{r}))$ & $R(=H(I T P$, UTPSND,$r)$ \\
\hline${ }_{3-7}^{1}$ & CMD $=S E N D$ & $\begin{aligned} \mathrm{U} 1= & =\mathrm{H} 0(\mathrm{u}, \mathrm{ITP}, \mathrm{r}) \\
\mathrm{U} 2=\mathrm{LIST} \text { members } & \\
& \text { addressed to } \mathrm{r}\end{aligned}$ & $\begin{array}{l}R 1=H O(I T P, U T P S N D, r) \\
R 2=R 1\end{array}$ \\
\hline 8 & Tabort & $\mathrm{U} 3=\mathrm{U} 1+\mathrm{U} 2$ & $\mathrm{R} 3=\mathrm{R} 2$ \\
\hline 11 & LIST $>$ empty & $\mathrm{U} 4=\mathrm{U} 33$ & $R 4=R 3$ \\
\hline 12 & & $\mathrm{US}=\mathrm{U} 4$ & $\mathrm{R} 5=\mathrm{R} 4$ +head (U2) \\
\hline 13 & & $\mathrm{v} 6=\mathrm{U5}$ & $R 6=R 5$ \\
\hline 11 & LIST $=$ empty & $U 7=U 6$ & $R 7=R 6$ \\
\hline 12 & & $\mathrm{UB}=\mathrm{U} 7$ & $\begin{array}{l}R 8=R 7 \\
R=R 2\end{array}$ \\
\hline 13 & & $v 9=48$ & $\mathbf{R} 9=\mathbf{R} 8$ \\
\hline
\end{tabular}

derivations:

$\mathrm{Un}=\ldots=\mathrm{U} 3=\mathrm{HO}(\mathrm{u}, \mathrm{ITP}, \mathrm{r})+$ LIST members addressed to $\mathrm{r}$

$R n=\ldots=R 1+$ head $(\operatorname{tail}(\operatorname{tail} \ldots($ U2) $))+\ldots+$ head(tall(U2)) + head(U2 $=R 1+U 2=H O(I T P, U T P S N D, r)+$ LIST members addressed to $r$,

path function: $H(u, I T P, r)=H($ ITP, UTPSND, $r)$

This, in conjunction with the UTP invariance then yields the intended function $\mathbf{f}$.

III. path 3 and 4 (-SEND)

\begin{tabular}{clll} 
part & condition & $\mathrm{U}(=\mathrm{H}(\mathrm{u}, \mathrm{ITP}, \mathrm{r}))$ & $\mathrm{R}(=\mathrm{H}(\mathrm{ITP}, \mathrm{UTPSND}, \mathrm{r})$ \\
\hline 1 & -(OPT=send) & $\begin{array}{l}\mathrm{U} 1=\mathrm{HO}(\mathrm{u}, \mathrm{ITP}, \mathrm{r}) \\
\mathrm{U} 2=\mathrm{U} 1 \mathrm{~N}\end{array}$ & $\begin{array}{l}\mathrm{R} 1=\mathrm{H} 0(\mathrm{ITP}, \mathrm{UTPSND}, \mathrm{r}) \\
\mathrm{R} 2=\mathrm{R} 1\end{array}$
\end{tabular}

derivations: $\quad \mathrm{U}_{2}=\mathrm{HO}(\mathrm{u}, \mathrm{ITP}, \mathrm{r}), \mathrm{R2}=\mathrm{HO}(\mathrm{ITP}, \mathrm{UTPSND}, \mathrm{r})$

This together with the UTP Invariance (g) leads directly to the intended function $f$.

pass

pass suff

This concludes our formal proof. Note that what has been proved was the "sufficient correctness" of ITP [14], since our invariant assertion says nothing about invalid SEND requests (requests addressed to invalid USERIDs or NODEIDs). 